\title{
Vineyard identification in an oak woodland landscape with airborne digital camera imagery
}

\author{
P. GONG †t, S. A. MAHLER $\ddagger$ G. S. BIGING $\ddagger$ and \\ D. A. NEWBURN
}

$\dagger$ International Institute for Earth System Science, Nanjing University, 210093, China; e-mail: gong@nature.berkeley.edu

tCenter for Assessment and Monitoring of Forest and Environmental Resources, 145 Mulford Hall, University of California, Berkeley, CA 94720-3114, USA

(Received 21 February 2001; in final form 9 October 2001)

\begin{abstract}
Using airborne multispectral digital camera imagery, we compared a number of feature combination techniques in image classification to distinguish vineyard from non-vineyard land-cover types in northern California. Image processing techniques were applied to raw images to generate feature images including grey level co-occurrence based texture measures, low pass and Laplacian filtering results, Gram-Schmidt orthogonalization, principal components, and normalized difference vegetation index (NDVI). We used the maximum likelihood classifier for image classification. Accuracy assessment is performed using digitized boundaries of the vineyard blocks. The most successful classification as determined by t-tests of the Kappa coefficients was achieved based on the use of a texture image of homogeneity obtained from the near infrared image band, NDVI and brightness generated through orthogonalization analysis. This method averaged an overall accuracy of 81 per cent for six frames of images tested. With post-classification morphological processing (clumping and sieving) the overall accuracy was significantly increased to 87 per cent (with a confidence level of 0.99).
\end{abstract}

\section{Introduction}

Accurate acreage estimates and mapping of California vineyards is of great interest to vineyard owners, wineries, environmentalists and land-use planners. According to the 'California Grape Acreage' report, California state-wide wine grape acreage nearly doubled from 1990 to 1998 (California Agricultural Statistics Service (CASS) 1999). Counties with the most rapid growth in vineyard development often have the least reliable acreage statistics (Heaton and Merenlender 2000). Furthermore, data published by CASS at the county level are not site-specific. Improved geographical precision and maps of current and historical vineyard acreage data would benefit land-use studies and wine industry strategic planning. A timely mapping of vineyard distribution can only be done with the use of remote sensing.

Few studies have been reported on the use of remote sensing to identify vineyards. Trolier et al. (1989) reported a failure in the State of New York when using Landsat Thematic Mapper imagery in the summer to distinguish vineyards from other vegetation cover types with a maximum likelihood classification algorithm. Grierson (2000)

International Journal of Remote Sensing

ISSN 0143-1161 print/ISSN 1366-5901 online (C) 2003 Taylor \& Francis Ltd

http://www tandf.co.uk/journals DOI: $10.1080 / 01431160110115870$ 
reported no significantly different spectral changes between two types of grape under different water conditions with data collected in South Australia with an 8-band radiometer between $460-813 \mathrm{~nm}$.

Vine canopy densities vary due to stress (water and disease), vine age, and vine spacing. There may be portions of a vineyard that are so young or stressed that they are unlikely to be classified as vineyard; however, from a user point of view these areas would ideally be included in total vineyard acreage since the land-use is likely to remain vineyard. The same situation holds for unplanted blocks. Although unplanted blocks are likely to be classified as non-vineyard, certain applications may prefer that these blocks be included in the vineyard land-use class. In Sonoma and Napa counties, it is likely that unplanted blocks will eventually be replanted to vineyard due to very high land value. In this paper we report the use of airborne multispectral digital camera imagery with approximately $2 \mathrm{~m}$ ground resolution in the identification of vineyards in Northern California. We estimate vineyard acreage regardless of vineyard status or health.

\section{Methods}

2.1. Study area

The study area includes images from Napa and Sonoma Counties. The primary land-cover types are vineyards and oak woodlands. Uncultivated landscape features in this Mediterranean climate are very dry in the summer. For example the hillside grasses tend to have a golden brown colour, while vigorous vines are in green. The proportion and shape of the vineyard and non-vineyard areas in the images vary, as does the topography. Some of the images contain large, rectangular vineyard blocks positioned primarily on the valley floor, and some contain irregularly shaped vineyard blocks in the hills. The terrain variation is important in the accuracy assessment of classification techniques since some methods will perform better than others in different terrain types due to sun angle and differences in natural vegetation with altitude.

\subsection{Image data and training area selection}

Aerial imagery was acquired on 31 July 1997 by Positive Systems, Inc., Whitefish, Montana, with their manufactured ADAR aerial imaging system. The ADAR sensor is composed of a series of four monochrome Kodak DCS 420 digital cameras. Each camera collects data for one band (blue, green, red, or near infrared). The resulting four bands are registered together to form an image with four co-registered bands. The spectral range of each band is listed in table 1.

We classified six ADAR images. Two images from different terrain types are used as examples throughout the paper (listed as images 2 and 6 in the results). Figure 1(a)

Table 1. TM and ADAR spectral bands.

\begin{tabular}{lccl}
\hline Band & $\begin{array}{c}\text { TM spectral range } \\
(\mu \mathrm{m})\end{array}$ & $\begin{array}{c}\text { ADAR spectral range } \\
(\mu \mathrm{m})\end{array}$ & Colour \\
\hline 1 & $0.45-0.52$ & $0.45-0.54$ & Blue \\
2 & $0.52-0.60$ & $0.52-0.60$ & Green \\
3 & $0.63-0.69$ & $0.61-0.68$ & Red \\
4 & $0.76-0.90$ & $0.78-1.00$ & Near infrared \\
\hline
\end{tabular}




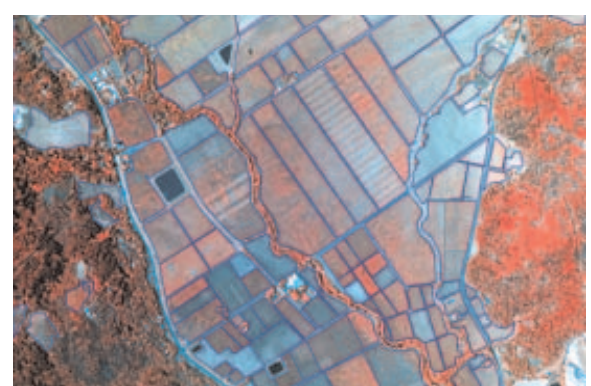

(a)

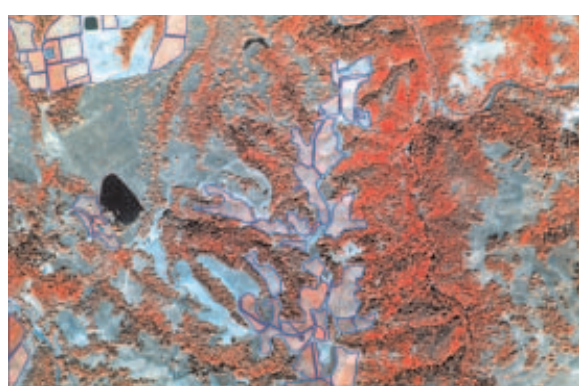

(b)

Figure 1. Standard false colour composite. (a) Image 2, (b) image 6.

centers on valley floor vineyard blocks surrounded by hills with some hillside vineyard blocks. Figure $1(b)$ centers on a hillside vineyard, with some valley floor blocks in the upper left corner. The blue lines are the digitized vineyard blocks used for accuracy assessment.

Training areas for supervised classification were created for each ADAR image based on image interpretation for 10 cover classes (table 2). Each of the six ADAR images has a unique set of training data created specifically for that image. Training areas were selected at the pixel level and included at least 200 pixels per class, and were distributed throughout the image to the extent that the features allowed.

The strategy for selecting training areas was to establish spectrally distinct cover classes for classification. Training class selection was based on image interpretation of a colour infrared image consisting of near infrared, red and green bands. We divided vines into three categories: high vigour vines, which have bright red pixels with solid canopy closure; medium vigour vines, which have medium intensity red and moderate to solid canopy closure; and low vigour vines, which have pink to light red pixels with low to moderate canopy closure. In post-classification processing the 10 cover classes were grouped into two classes, vineyard and non-vineyard. Accuracy assessment of the classification schemes is performed on these 2-class images.

Table 2. ADAR training area cover classes.

\begin{tabular}{ll}
\hline Classification cover class & Post-classification cover class \\
\hline Vine-high vigour & Vine \\
Vine-medium vigour & Vine \\
Vine-low vigour & Vine \\
Grass-light & Non-vine \\
rass-dark & Non-vine \\
Soil & Non-vine \\
Water & Non-vine \\
Tree-high vigour & Non-vine \\
Tree-medium vigour & Non-vine \\
Tree-shade & Non-vine \\
\hline
\end{tabular}




\subsection{Feature image generation}

2.3.1. Gram-Schmidt orthogonalization technique

Linear combinations of spectral bands can be used to form physically significant indices (Jackson 1983). One example is the tasselled cap transformation (Kauth and Thomas 1976, Crist and Kauth 1986). Tasselled cap coefficients were developed for Landsat TM and MSS imagery only. We analysed the six ADAR images using the Gram-Schmidt orthogonalization technique to calculate coefficients for greenness, brightness and yellowness (Jackson 1983). As in the Kauth and Thomas transformation, greenness represents the abundance and vigour of living vegetation while brightness represents soil condition (Campbell 1996). Yellowness is not representative of recognizable landscape features, and is not used in this research.

Calculation of the coefficients for greenness, brightness and yellowness was based on mean grey level values of the raw imagery of four cover types. Through examination of 2D scatterplots of infrared and red images we collected grey level values from suitable areas for coefficient calculation. Water was used to represent wet soil because very wet soils are rare in the imagery. Dry soil was selected from both dirt roads and exposed soil. Tree canopies were found to be on the most extreme portion of the vegetation line. Due to the Mediterranean climate natural grasses are typically dried out in the summer, and are representative of the senesced vegetation that lies on the extreme low end of the vegetation line in the project scenes. The grey level values for the four classes averaged for all six ADAR images are listed in table 3 .

The Gram-Schmidt coefficients were calculated separately for each ADAR image, and table 4 lists these coefficients. The average of these coefficients was used to create brightness, greenness and yellowness bands.

Table 3. Mean grey level values for sampled land areas in the six ADAR images.

\begin{tabular}{lrrrr}
\hline Cover type & Band 1 & Band 2 & Band 3 & Band 4 \\
\hline Wet soil & 90 & 76 & 77 & 66 \\
Dry soil & 175 & 176 & 207 & 159 \\
Green vegetation & 98 & 90 & 92 & 171 \\
Senesced vegetation & 144 & 139 & 167 & 139 \\
\hline
\end{tabular}

Table 4. Calculated Gram-Schmidt band coefficients for the six ADAR images.

\begin{tabular}{|c|c|c|c|c|c|c|c|c|c|c|c|c|}
\hline \multirow[b]{2}{*}{ Image } & \multicolumn{4}{|c|}{ Brightness } & \multicolumn{4}{|c|}{ Greenness } & \multicolumn{4}{|c|}{ Yellowness } \\
\hline & Blue & Green & Red & NIR & Blue & Green & Red & NIR & Blue & Green & Red & NIR \\
\hline 1 & 0.37 & 0.47 & 0.66 & 0.46 & -0.44 & -0.51 & -0.74 & 0.05 & -0.41 & -0.56 & -0.66 & -0.30 \\
\hline 2 & 0.45 & 0.51 & 0.61 & 0.40 & -0.50 & -0.55 & -0.67 & 0.02 & -0.41 & -0.56 & -0.66 & -0.30 \\
\hline 3 & 0.41 & 0.47 & 0.62 & 0.48 & -0.48 & -0.53 & -0.69 & 0.06 & -0.44 & -0.52 & -0.59 & -0.44 \\
\hline 4 & 0.42 & 0.48 & 0.62 & 0.44 & -0.47 & -0.53 & -0.70 & 0.07 & -0.45 & -0.56 & -0.61 & -0.32 \\
\hline 5 & 0.38 & 0.46 & 0.63 & 0.50 & -0.47 & -0.52 & -0.71 & 0.10 & -0.47 & -0.55 & -0.62 & -0.30 \\
\hline 6 & 0.44 & 0.50 & 0.60 & 0.45 & -0.48 & -0.51 & -0.70 & 0.14 & -0.48 & -0.54 & -0.63 & -0.28 \\
\hline Average & 0.41 & 0.48 & 0.62 & 0.45 & -0.47 & -0.53 & -0.70 & 0.07 & -0.44 & -0.55 & -0.63 & -0.32 \\
\hline $\begin{array}{l}\text { standard } \\
\text { deviation }\end{array}$ & 0.03 & 0.02 & 0.02 & 0.03 & 0.02 & 0.02 & 0.02 & 0.04 & 0.03 & 0.01 & 0.03 & 0.06 \\
\hline
\end{tabular}


The resulting equations for greenness, brightness and yellowness are:

$$
\begin{aligned}
& \text { Greenness }=-0.47(\text { Blue })-0.53(\text { Green })-0.70(\text { Red })+0.07(\mathrm{NIR}) \\
& \text { Brightness }=0.41(\text { Blue })+0.48(\text { Green })+0.62(\text { Red })+0.45(\text { NIR }) \\
& \text { Yellowness }=0.44(\text { Blue })-0.55(\text { Green })-0.63(\text { Red })-0.32(\mathrm{NIR})
\end{aligned}
$$

Figures $2(a)$ and $2(b)$ depict the resulting brightness and greenness bands for image 2. The highest values in the brightness image are representative of dry soil and are shown in white. For example, the dirt roads between the vineyard blocks appear white in figure $2(a)$. Trees have the highest value in the greenness image, as seen on the left side of figure $2(b)$.

\subsubsection{Co-occurrence texture measures}

The spatial arrangement of spectral features can be used to help distinguish cover classes in the ADAR images using texture measures. Texture is a measure of the spatial and spectral relationships between neighbouring pixels (Campbell 1996). Co-occurrence texture measures use a grey level spatial dependence matrix to calculate texture values. This matrix contains relative frequencies of pixel values that occur in two neighbouring pixels separated by a specified distance and direction with a kernel of certain size. The matrix enumerates the number of occurrences of the relationship between a pixel and its specified neighbour (Gong et al. 1992).

Several co-occurrence texture measures were initially examined in this study including mean, variance, homogeneity, contrast, dissimilarity, entropy, second moment, and correlation. Homogeneity provided the best and most consistent results for the six images here. Of the four raw bands we determined through experimentation that texture measures applied to the infrared band produced the best results for distinguishing vineyard from non-vineyard classes. Through visual examination of several kernel sizes we determined 15 by 15 to be the best.

\subsection{3. $P C A$}

Principal component analysis (PCA) uses a linear transformation of multi-band data to translate and rotate data into a new coordinate system that removes correlation between the axes. This technique reduces the dimensionality of multi-band image by generating one or several bands that contains most of the data variability (Gong and Howarth 1990a). Several kernel sizes were applied to the first principal

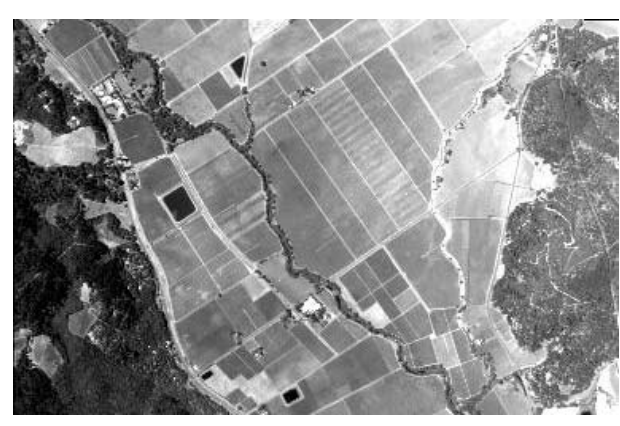

(a)

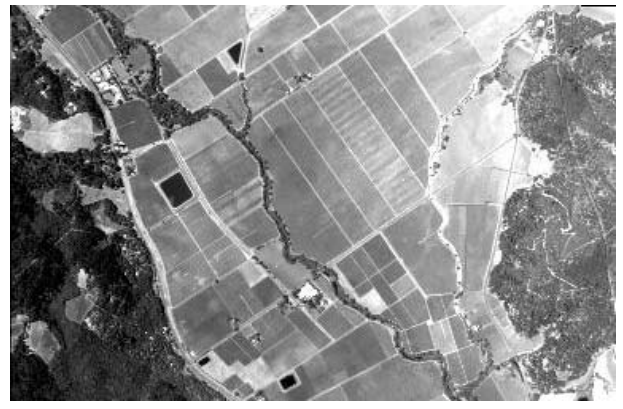

(b)

Figure 2. Gram-Schmidt orthogonalization results from image 2. (a) Brightness image, (b) greenness image. 
component (PC1). Analysis of Kappa coefficients derived from preliminary classifications using these various kernel sizes lead us to choose 15 by 15 (figure 3 ). The $15 \times 15$ homogeneity filter of PC1 will be abbreviated 15 HPC1.

\subsubsection{High pass Laplacian and low pass filters}

A Laplacian filter was applied to the NIR imagery. Through visual assessment of several filter sizes $(5 \times 5,7 \times 7$ and $9 \times 9)$, we found a $7 \times 7$ Laplacian filter was the most suitable in distinguishing the tree canopies from other cover types. However, this filter produces edges with speckling effects. A smoothing can be applied to the edge images to generate edge density images for classification purposes (Gong and Howarth 1990b). Through visual examination of several filter sizes $(7 \times 7,9 \times 9$, $15 \times 15,21 \times 21,29 \times 29$ and $35 \times 35$ ) we used a $29 \times 29$ filter size. The resulting images of a $29 \times 29$ low pass filter applied to a $7 \times 7$ Laplacian filter of the NIR band was referred to as 29LP7L. The resulting feature images for images 2 and 6 are presented in figure 4.

Low pass filters improve on the ability of the Laplacian filter in distinguishing the trees from the other cover classes. We grouped greenness, brightness and the 29LP7L band for the fourth combination of feature images.

For the final band combination of feature images a $15 \times 15$ homogeneity texture image was derived from the NIR band (figure 5).

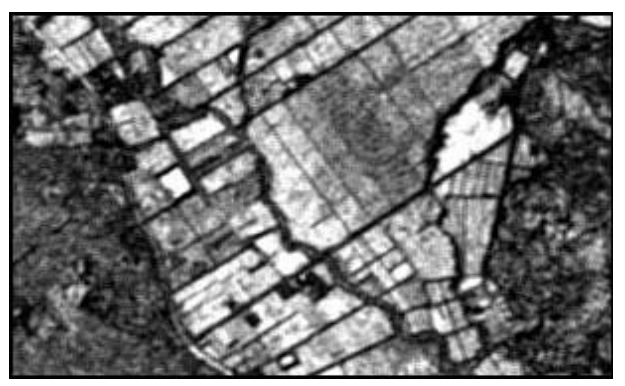

(a)

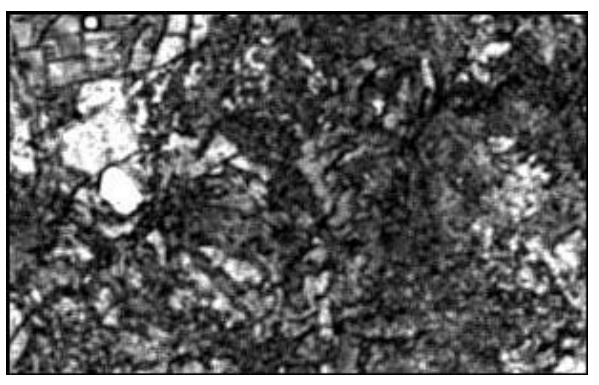

(b)

Figure 3. Homogeneity of PC-1 with a kernel size of $15 \times 15$. (a) Image 2, (b) image 6 .

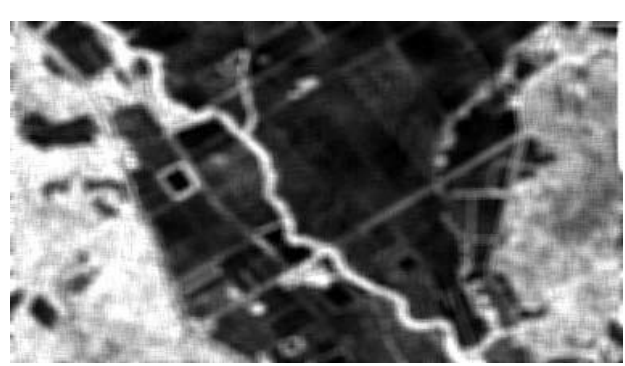

(a)

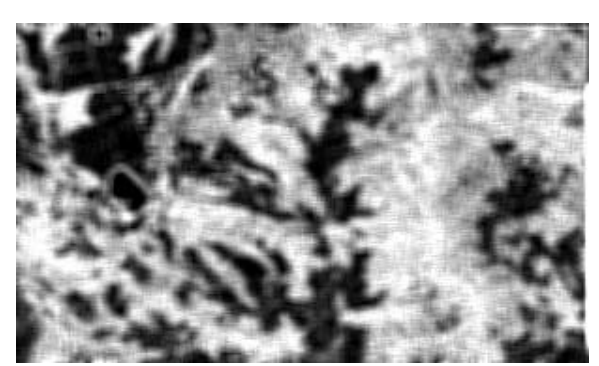

(b)

Figure 4. (a) Laplacian applied to NIR image 2 then smoothed (29LP7LNIR), (b) Laplacian applied to NIR image 6 then smoothed (29LP7LNIR). 


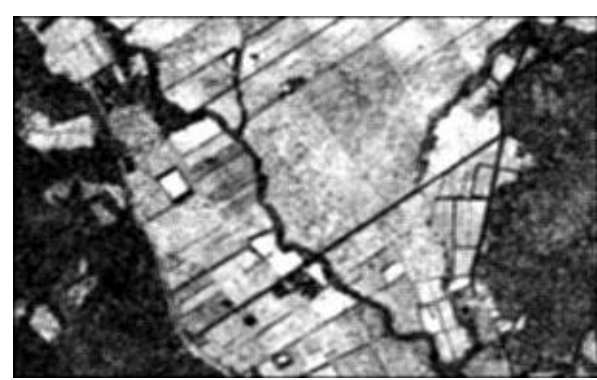

(a)

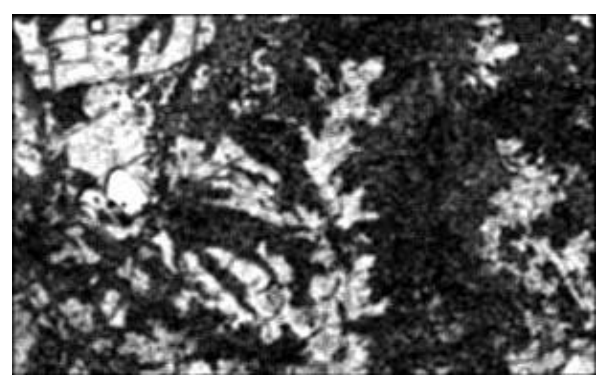

(b)

Figure 5. Homogeneity texture images 15HIR. (a) Image 2, (b) image 6.

\subsection{Post-classification processing}

\subsubsection{Sieve and clump classes}

Many of the vine errors of commission are isolated clusters of pixels. Postclassification processing is performed to remove these errors from the classified image. We used 'sieving' and then 'clumping' for reducing the level of commission.

Sieving looks at the neighbouring four or eight pixels to determine if a pixel is grouped with pixels of the same class. Similar to sifting soil through a sieve to retain the rocks, the groups of adjacent pixels in a cover class smaller than that defined in the sieve process are removed and replaced with unclassified pixels. Sieving will leave speckles or holes in the classified image of unclassified pixels. Clumping can be performed after sieving to replace the unclassified pixels into the class of the adjacent pixels. The selected classes are clumped together by applying a morphological filter to the sieved classification data by first performing a dilate operation and then an erode operation on the classified image using a kernel of specific size (Research Systems, Inc. 2000).

With post-classification processing some correctly classified pixels will be inappropriately changed. For example, weak vineyard blocks may have clumps of diseased or dead vines, or absent vines that will be inappropriately changed to unclassified pixels. Additionally, properly classified non-vine pixels surrounded by incorrectly classified vine pixels may be inappropriately changed. A balance between commission and omission can be obtained through experiment.

\subsection{Accuracy assessment}

Accuracy assessment was performed using reference images that consist of two classes, vineyard and non-vineyard. Reference images were created using interpretation results of the raw images by one of the coauthors, who made a number of field visits to the study area. Masks were digitized using vineyard blocks in each image. Examples of the digitized boundaries are shown in figure 1; the vineyard blocks are delineated in purple. Pixels within the digitized vineyard blocks were considered to be vineyard, while pixels outside those boundaries non-vineyard. Classified images were compared pixel by pixel with the reference images to derive the overall accuracies and kappa coefficients of various classification methods.

In order to determine the impact of weak and possibly unplanted vineyard blocks, we divided the digitized vineyard blocks into high, medium and low vigour. Vineyard blocks were not rigorously defined; they were identified and categorized through image interpretation. Some of those blocks appeared to be soil. Because of their 
shape and proximity to medium and high vigour vineyard blocks we could state with confidence that the low vigour blocks were, are, or would become vineyard blocks. Some vineyard blocks are consistently vigorous and healthy, while others are patchy with some areas vigorous and other areas bare ground. Therefore, the vigour class assignment of the digitized boundaries was determined by the dominant status of the vines in a given block. Figure 6 shows examples of the digitized blocks. High vigour digitized vineyard block boundaries in red, medium vigour in purple and low vigour in yellow.

In the original method of accuracy assessment, all digitized vineyard blocks are included in the vineyard class in the two-class reference images. In the alternative classification accuracy assessments, the low vigour vines are moved from vineyard to the non-vineyard class in the reference image; only the high and medium vigour blocks are used in the vineyard class. The original method provides a better assessment of the various classification methods' ability to map land-use. The alternative method provides a comparison of classification performance of the two methods.

\section{Results}

Results for the classification methods are listed in tables 7-9. Method 1 is the classification of the four raw data bands. Methods 2-7 represent the six combination classification schemes. Overall, methods 2,3 and 4 produced very similar results, while methods 5, 6 and 7 produced similar results that were superior to methods 2, 3 and 4 . Methods $5 \mathrm{~b}, 5 \mathrm{~b} 2$, and $7 \mathrm{~b}$ are the post-classification sieve and clump processes applied to methods 5 and 7, respectively. Method 7c is the same as method 7 except that the low vigour vineyard boundaries are considered to be non-vine. These methods are listed below.

1. Raw ADAR bands.

2. Greenness, brightness, homogeneity (kernel $15 \times 15$ ) of principle component 1 .

3. NDVI, brightness, homogeneity (kernel $15 \times 15$ ) of principle component 1 .

4. IR, greenness, homogeneity (kernel $15 \times 15)$ of principle component 1 .

5. NDVI, brightness, low pass filter $(15 \times 15)$ applied to a homogeneity (kernel $15 \times 15$ ) of the IR band.

6. NDVI, brightness, low pass filter $(29 \times 29)$ of a Laplacian filter $(7 \times 7)$ applied to the IR band.

7. Greenness, brightness, low pass filter $(29 \times 29)$ of a Laplacian filter $(7 \times 7)$ applied to the IR band.

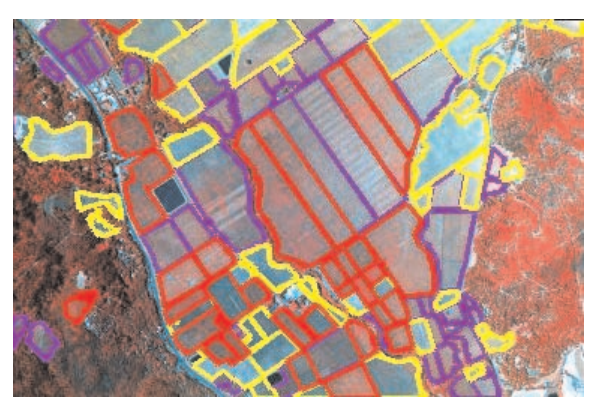

(a)

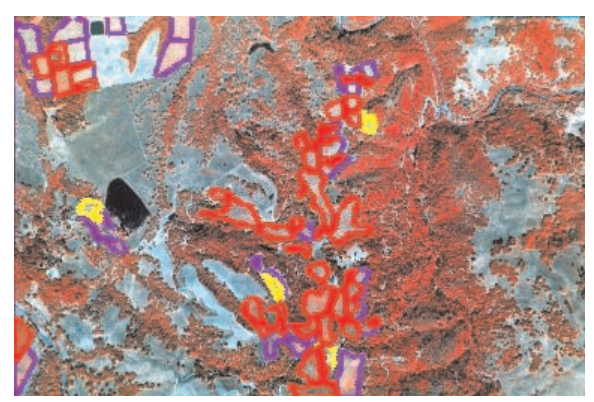

(b)

Figure 6. Digitized blocks of different vineyard types for (a) image 2 and (b) image 6. 
5b. Sieve/clump (196 pixels) of vine and non-vine classes applied to method 5 .

5 b2. Same as method 5 b, except that the low vigour vineyard boundaries are considered non-vine.

7b. Sieve/clump (196 pixels) of vine and non-vine classes applied to method 7.

7c. Same as method 7, except that the low vigour vineyard boundaries are considered non-vine.

Table 5 lists confusion matrices in percentages for vine and non-vine, errors of commission and omission. Data are presented as an average of all six images. Because vineyard land occupies less area than non-vine land in all images, particularly image 4 , the errors of commission for vine are high. When image 4 is excluded, the errors of commission for vine have dropped considerably (as shown in brackets of table 5). Table 6 lists overall accuracy results and table 7 lists the Kappa coefficients all derived from table 5 .

Variance for the Kappa coefficients was derived for all of the classification methods using equations in Congalton and Green (1999). A $Z$ statistic can be calculated to determine significant differences between pairs of classifications. Because there are so many permutations of classification pairs, the methods were assessed in groupings. All tests of significance have a confidence level of 0.99. Calculation for two independent error matrices (equation 1) is presented below. Results of these tests are listed in table 8.

$$
Z=\left|K_{1}-K_{2}\right| /\left(\left(\operatorname{var}\left(K_{1}\right)+\operatorname{var}\left(K_{2}\right)\right)^{1 / 2}\right)
$$

First we performed $t$-tests for classification methods 2,3 and 4 to determine if

Table 5. Confusion matrices with errors of commission and omission.*

\begin{tabular}{llcccc}
\hline Method & Class & $\begin{array}{c}\text { Ground truth } \\
\text { vine }\end{array}$ & $\begin{array}{c}\text { Ground truth } \\
\text { non-vine }\end{array}$ & $\begin{array}{c}\text { Errors of } \\
\text { commission }\end{array}$ & $\begin{array}{c}\text { Errors of } \\
\text { commission }\end{array}$ \\
\hline 1 & Vine & 77 & 29 & $192(96)$ & 23 \\
& Non-vine & 23 & 71 & 10 & 29 \\
2 & Vine & 70 & 18 & $141(56)$ & 28 \\
& Non-vine & 28 & 82 & 12 & 18 \\
3 & Vine & 74 & 20 & $158(60)$ & 26 \\
& Non-vine & 26 & 80 & 11 & 20 \\
4 & Vine & 71 & 18 & $146(64)$ & 29 \\
& Non-vine & 29 & 82 & 12 & 21 \\
5 & Vine & 74 & 14 & $121(34)$ & 26 \\
& Non-vine & 26 & 86 & 11 & 14 \\
6 & Vine & 76 & 16 & $147(41)$ & 24 \\
& Non-vine & 24 & 84 & 10 & 16 \\
7 & Vine & 74 & 14 & $121(36)$ & 26 \\
& Non-vine & 26 & 86 & 11 & 14 \\
$5 \mathrm{~b}$ & Vine & 69 & 6 & $63(8)$ & 31 \\
& Non-vine & 31 & 94 & 13 & 6 \\
$5 \mathrm{~b} 2$ & Vine & 80 & 7 & $87(18)$ & 20 \\
& Non-vine & 20 & 93 & 5 & 7 \\
$7 \mathrm{~b}$ & Vine & 68 & 7 & $43(7)$ & 32 \\
& Non-vine & 32 & 96 & 13 & 4 \\
$7 \mathrm{c}$ & Vine & 80 & 6 & $63(17)$ & 20 \\
& Non-vine & 21 & 94 & 5 & 6 \\
\hline
\end{tabular}

*Numbers in brackets are calculated by excluding image 4 . 
Table 6. Overall classification accuracy of combination methods $1-7 \mathrm{c}$.

\begin{tabular}{lccccccccccc}
\hline Image & 1 & 2 & 3 & 4 & 5 & 6 & 7 & $5 b$ & $5 b 2$ & $7 b$ & $7 \mathrm{c}$ \\
\hline 1 & 69 & 78 & 75 & 78 & 79 & 72 & 77 & 87 & 92 & 86 & 91 \\
2 & 72 & 77 & 76 & 76 & 78 & 82 & 82 & 81 & 89 & 82 & 88 \\
3 & 74 & 78 & 80 & 79 & 78 & 82 & 81 & 86 & 90 & 89 & 93 \\
4 & 64 & 70 & 65 & 72 & 68 & 64 & 71 & 82 & 81 & 88 & 87 \\
5 & 70 & 82 & 82 & 82 & 89 & 88 & 87 & 89 & 92 & 90 & 93 \\
6 & 81 & 86 & 85 & 85 & 95 & 94 & 93 & 96 & 97 & 96 & 96 \\
Average & 72 & 78 & 77 & 79 & 81 & 80 & 82 & 87 & 90 & 89 & 91 \\
\hline
\end{tabular}

Table 7. Kappa coefficient for classification methods 1-7c.

\begin{tabular}{|c|c|c|c|c|c|c|c|c|c|c|c|}
\hline Image & 1 & 2 & 3 & 4 & 5 & 6 & 7 & $5 b$ & $5 \mathrm{~b} 2$ & $7 b$ & $7 \mathrm{c}$ \\
\hline 1 & 27 & 36 & 32 & 36 & 41 & 32 & 38 & 54 & 66 & 54 & 64 \\
\hline 2 & 44 & 53 & 52 & 53 & 57 & 65 & 65 & 62 & 76 & 64 & 75 \\
\hline 3 & 42 & 48 & 52 & 49 & 48 & 55 & 53 & 65 & 71 & 69 & 78 \\
\hline 4 & 13 & 17 & 14 & 18 & 16 & 13 & 17 & 29 & 25 & 38 & 34 \\
\hline 5 & 35 & 56 & 56 & 55 & 70 & 69 & 67 & 71 & 77 & 74 & 78 \\
\hline 6 & 38 & 43 & 44 & 42 & 71 & 69 & 66 & 78 & 79 & 75 & 76 \\
\hline Average & 33 & 42 & 42 & 42 & 51 & 50 & 51 & 60 & 66 & 62 & 68 \\
\hline
\end{tabular}

Table 8. Significant differences between classification methods.

\begin{tabular}{lccccc}
\hline Image & $\begin{array}{c}\text { Methods 2, } \\
3,4\end{array}$ & $\begin{array}{c}\text { Methods 5, } \\
6,7\end{array}$ & $\begin{array}{c}\text { Methods } 1, \\
2,5\end{array}$ & $\begin{array}{c}\text { Methods 5, 5b, } \\
5 \mathrm{~b} 2\end{array}$ & $\begin{array}{c}\text { Methods } 7,7 \mathrm{~b}, \\
\mathrm{c}\end{array}$ \\
\hline 1 & $2>4>3$ & $5>7>6$ & $5>2>1$ & $5 \mathrm{~b} 2>5 \mathrm{~b}>5$ & $7 \mathrm{~b}>7 \mathrm{c}>7$ \\
2 & $2>4>3$ & $7=6>5$ & $5>2>1$ & $5 \mathrm{~b} 2>5 \mathrm{~b}>5$ & $7 \mathrm{c}>7>7 \mathrm{~b}$ \\
3 & $4>2>3$ & $7>5>6$ & $2>5>1$ & $5 \mathrm{~b}>5 \mathrm{~b} 2>5$ & $7 \mathrm{~b}>7>7 \mathrm{c}$ \\
4 & $2>4=3$ & $5>6>7$ & $5>2>1$ & $5 \mathrm{~b} 2>5 \mathrm{~b}>5$ & $7 \mathrm{~b}>7 \mathrm{c}>7$ \\
5 & $3>2>4$ & $6>7>5$ & $5>2>1$ & $5 \mathrm{~b} 2>5 \mathrm{~b}>5$ & $7 \mathrm{~b}>7 \mathrm{c}>7$ \\
6 & $3>2>4$ & $5>6>7$ & $5>2>1$ & $5 \mathrm{~b} 2>5 \mathrm{~b}>5$ & $7 \mathrm{~b}>7 \mathrm{c}>7$ \\
\hline
\end{tabular}

significant differences exist. All three of these methods utilized texture (homogeneity) of the first principle component. The other two bands used in these classifications were NDVI and brightness for method 2, IR and greenness for method 3 and IR and greenness for method 4. Method 2 was significantly better than the other two methods for three of the six study images; method 3 was significantly better for two of the images, and method 4 was significantly better for one image. While there are not enough study images to select the best method of these three methods, we choose method 2 for further comparisons since it produced the best results in the majority of the six study images.

We then performed t-tests for classification methods 5, 6 and 7, which also produced mixed results. These methods use low pass filters on either homogeneity or Laplacian filters of the IR band. The other two bands used in these classifications are NDVI and brightness for methods 5 and 6, and greenness and brightness for method 7. Method 5 was significantly better than the other two methods for three of the study images. There was no clearly 'best' method of these three, but we used 
method 5 for further comparison since it was significantly better than the others in the majority of the images.

We compared methods 2, 5 and 1 . Methods 2 and 5 were significantly better than method 1 for all six images. Therefore, the generated bands improved upon the raw data classification. For five of the six study images method 5 was significantly better than method 2 . There was one image for which method 2 was significantly better than method 5. This suggests that homogeneity of the IR band is more effective than homogeneity of the first principle component at distinguishing vine and non-vine classes.

Methods $5,5 \mathrm{~b}$ and $5 \mathrm{~b} 2$ were also assessed. Method $5 \mathrm{~b}$ is the application of clumping and sieving to the results of method 5. In all cases, method $5 \mathrm{~b}$ was significantly better than method 5 . Therefore, the clumping and sieving process applied to method 5 improved the classification results. Method $5 \mathrm{~b} 2$ is the same as method $5 \mathrm{~b}$ except that low vigour vine blocks are considered to be non-vine in method $5 \mathrm{~b} 2$. In five of the six study images method $5 \mathrm{~b} 2$ was significantly better than method $5 \mathrm{~b}$. This suggests poor classification of low vigour vineyard blocks.

The final grouping of classification methods assessed with t-tests are methods 7 , $7 \mathrm{~b}$ and $7 \mathrm{c}$. Method $7 \mathrm{~b}$ applies sieving and clumping to method 7. Method $7 \mathrm{c}$ is the same as method 7 except that low vigour vineyard blocks are considered to be nonvine. Method $7 b$ is better than method 7 in all but one of the study images, therefore, the clumping and sieving post-classification process is an effective method of improving classification accuracy for method 7. Method 7c is better than method 7 in all but one of the study images, suggesting again that the classification of low vigour vineyard blocks is poor.

Of the six feature combination methods $(2,3,4,5,6$, and 7) method 5 had the best overall performance. In all but one case the use of the sieve/clump process for methods 5 and 7 significantly improved the accuracies. As discussed previously, errors of omission for vine are expected due to the fact that there are many weak, non-bearing, or unplanted vineyard blocks that are digitized and counted as vineyard in the reference images, but unlikely to be classified as vineyard. Therefore, accuracy results for $5 \mathrm{~b} 2$ and $7 \mathrm{c}$ are listed as a reference to examine what the classification accuracy would be if we did not include these blocks in the reference image as vine. In all but one of 12 cases, the omission of low vigour vineyard blocks from the vine class significantly improved the Kappa coefficient. This suggests a better method for distinguishing these low vigour vineyard blocks is necessary.

The sieve and clump procedures used in methods $5 \mathrm{~b}$ and $7 \mathrm{~b}$ greatly reduced errors of commission for vine but slightly increased vine errors of omission. Errors of commission for non-vine were increased slightly in methods $5 \mathrm{~b}$ and $7 \mathrm{~b}$ (from methods 5 and 7), while non-vine errors of omission were reduced by more than half. Errors of omission for vine go up by approximately one fifth for the sieve/clump methods. None of the methods improve the maximum likelihood classification results for the raw data for errors of omission for vine (except methods $5 \mathrm{~b} 2$ and $7 \mathrm{c}$, which exclude low vigour vines). All methods reduce errors of omission for non-vine as compared to the raw data classification. All methods improve upon the raw data maximum likelihood results for non-vine, however results for vine are not as good.

Figures $7(a)$ and $(b)$ depict classifications 5 and $5 b$ for images 2 and 6 , respectively. The exclusion of the low vigour blocks from the vineyard class in methods $5 \mathrm{~b} 2$ and 7c improves the producer's accuracy and user's accuracy in methods 5 and 7 . Vine commission errors are a very large source of errors when ADAR image 4 is included, and closer (or less than) vine errors of omission when ADAR image 4 is excluded. 


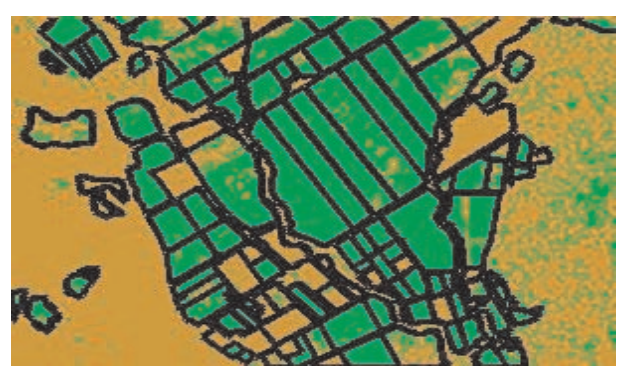

(a)

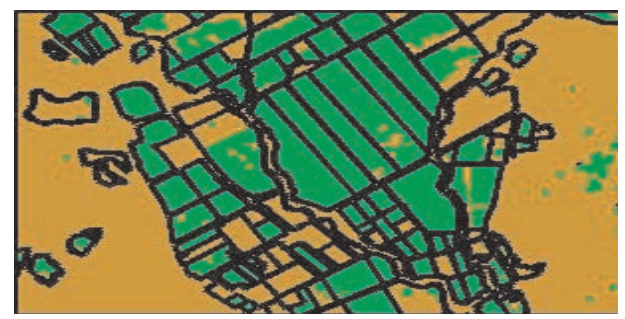

(c)

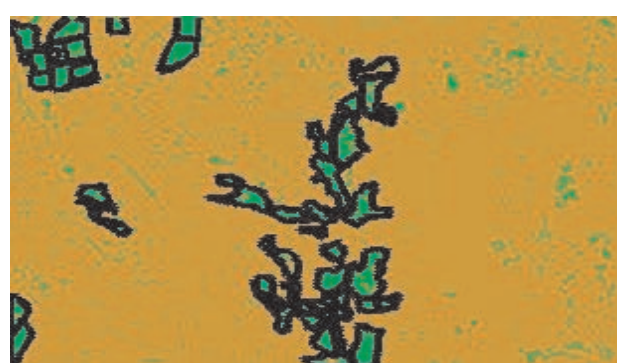

(b)

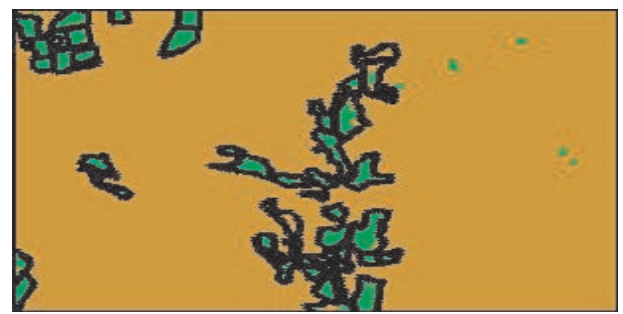

(d)

Figure 7. (a) Method 5 classification results for image 2, (b) method 5 classification results for image 6, $(c)$ method 5 b classification results for image 2, $(d)$ method 5 b classification results for image 6 .

\section{Discussion and summary}

The results of this research suggest that vineyards can be identified from $2 \mathrm{~m}$ resolution airborne multispectral digital camera imagery with an acceptable level of accuracy $(>85 \%)$. This must be done by using a combination of image spatial features with spectral features followed by post-classification processing. Since there are numerous spatial feature extraction methods and the number of spatial feature combinations is large, it is difficult to exhaust all combinations and achieve the best classification results. Thus there is room for further improvement. For example, the best combination of bands for the three classification methods that utilized homogeneity of PC1 included greenness and brightness as the other two features in the combination. Adjusting method 5 to use greenness instead of NDVI may produce better results.

This research tested only the feasibility of airborne multispectral digital camera imagery for identifying vineyards. To measure vineyard acreage at the county or regional level would require complete image coverage. This would require additional image processing such as image registration, orthorectification and finally mosaicing. Mosaiced aerial data can then be classified to allow for non-repetitive acreage estimates and geographic delineation of vineyards. Such classification results produced at the county level could be compared with county level data published by CASS. These data would be of interest to land-use planners, environmentalists, and the wine industry.

Spectral separability of the low vigour vineyard blocks from native grasses and soil seems to be low and this presents the greatest challenge for improvement of vineyard classification at a landscape level. The use of time series image data may provide a solution to the spectral confusion of these classes. In late winter, the 
natural grasses in California are very green from the winter rains while the vineyards are dormant. During the summer the natural grasses are dry and brown in colour; while the vineyard canopies are at a mature stage and are bright green. Measurement of differences in brightness values of the vineyard and non-vineyard cover classes at different seasons may provide improved results. Since most vine growing areas in the world are in the sunny climate zones with relatively dry summer seasons, good atmospheric conditions are usually possible for either airborne and satellite imaging. Although satellite imaging lacks the flexibility in overpass time that airborne imaging does and the atmospheric effects on satellite images are usually greater than airborne images, the most severely affected channels are the shorter wavelength channels such as the blue and green bands. Since our results are primarily relaying on the longer wavelength channels such as the red and infrared, the results obtained in this research have direct relevance to the use of high resolution satellite imagery such as IKONOS panchromatic and multispectral data which have $1 \mathrm{~m}$ and $4 \mathrm{~m}$ spatial resolutions, respectively.

\section{References}

California Agriculture Statistics Service, 1999, California Grape Acreage. www.nass.usda.gov/ca.

Campbell, J. B., 1996, Introduction to Remote Sensing, Second Edition, (New York: Guildford Publications).

Congalton, R. G., and Green, K., 1999, Assessing the Accuracy of Remotely Sensed Data: Principles and Practices (New York: Lewis Publishers).

Crist, E. P., and Kauth, R. J., 1986, The Tasseled Cap De-Mystified. Photogrammetric Engineering and Remote Sensing, 52, 81-86.

Gong, P., and Howarth, P. J., 1990a, An assessment of some factors influencing multispectral land-cover classification. Photogrammetric Engineering and Remote Sensing, 56, 597-603.

Gong, P., and Howarth, P. J., 1990b, The use of structural information for improving landcover classification accuracies at the rural-urban fringe. Photogrametric Engineering and Remote Sensing, 56, 67-73.

Gong, P., Marceau, D. J., and Howarth, P. J., 1992, A comparison of spatial feature extraction algorithms for land-use classification with SPOT HRV data. Remote Sensing of Environment, 40, 137-151.

GrIERSON, I., 2000, The effect of soil water management on the reflectance characteristics of vine (vitis vinifera) leaves and their monitoring by low level airborne video. Geocarto International, 15, 43-51.

Heaton, E., and Merenlender, A., 2000, Modeling vineyard expansion, potential habitat fragmentation. California Agriculture 54(3), 12-19.

JACKSON, R. D., 1983, Spectral Indices in n-Space. Remote Sensing of Environment, 13, 409-421.

Kauth, R. J., and Thomas, G. S., 1976, The tasseled cap - a graphic description of the spectral-temporal development of agricultural crops as seen by Landsat, Proceedings, Symposium on Machine Processing of Remotely Sensed Data, Laboratory for Applications of Remote Sensing, Pudure University, West Lafayette, IN, pp.41-51.

ReSEARCh Systems InC., 2000, ENVI User's Guide, Version 3.4. Boulder, CO 80301, USA.

Trolier, L. J., Philipson, W. R., and Philpot, W. D., 1989, Landsat TM analysis of vineyards in New York. International Journal of Remote Sensing, 10, 1277-1281. 